\title{
The role of photo-ionization in extreme ultraviolet ablation interactions
}

\author{
J. Lolley, G. Tallents
}

J. A. Lolley, G. J. Tallents, "The role of photo-ionization in extreme ultraviolet ablation interactions," Proc. SPIE 11886, International Conference on X-Ray Lasers 2020, 118860T (8 July 2021); doi: 10.1117/12.2592289 


\title{
The role of photo-ionization in extreme ultraviolet ablation interactions
}

\author{
J A Lolley, G J Tallents \\ York Plasma Institute, Department of Physics, University of York, York, YO10 5DD, UK
}

\begin{abstract}
In the extreme ultraviolet (EUV) regime, the photon energies are above the threshold for photo-ionization in all solid materials. We examine the absorption coefficients for inverse bremsstrahlung and photo-ionization and the contributions of these processes to EUV absorption under different ionization models. The Saha-Boltzmann ionization model is considered with and without continuum lowering, as well as a novel rate equation model including the photo-ionization transitions in population calculations. We show that photo-ionization plays a significant role early in EUV ablation of solid aluminium, but inverse bremsstrahlung absorption dominates once temperatures in the ablated plasma exceed more than a few electron volts.
\end{abstract}

Keywords: ablation, short-wavelength, EUV, laser, theory

\section{INTRODUCTION}

Laser-solid interactions in the $1 \times 10^{10} \mathrm{~W} \mathrm{~cm}^{-2}$ irradiance limit are well understood for optical wavelengths. This regime lies at the lower end of possible ablation interactions but just above the threshold - intensities easily achievable in laboratory conditions with commercially available light sources. Tabletop light sources capable of these intensities at extreme ultraviolet wavelengths are now available ${ }^{1 ; 2}$, and ablation at these short wavelengths has been demonstrated multiple times ${ }^{3 ; 4 ; 5}$. The increase in photon energy between optical light and EUV light requires the consideration of photo-ionization as an integral part of these interactions.

The extreme ultraviolet regime extends from roughly $10 \mathrm{~nm}$ to $100 \mathrm{~nm}(\approx 12 \mathrm{eV}$ to $120 \mathrm{eV})$. At these wavelengths, two key limits are reached. The first is that for at least the first ionization in most elements the photon energy exceeds the ionization energy, $\hbar \omega>E_{i o n}$, and photo-ionization can occur. The second is an increase in the critical density $n_{\text {crit }}$ to above solid density $n_{\text {sol }}, n_{\text {crit }}>n_{\text {solid }}$, for most materials, allowing us to consider the plasma as under-dense and therefore that absorption contributions from collective processes such as resonance absorption are negligible. These two changes are significant alterations to the standard ablation absorption model for optical wavelengths, which is dominated by inverse bremsstrahlung and resonance absorption. In this paper we consider the significance of photo-ionization absorption in EUV laser ablation of solid aluminium targets. The ionization of the plasma formed is modelled using a rate-equation approach and Saha-Boltzmann equilibrium. Continuum lowering of the ionization potentials is shown to have a small effect on the depths of ablated features.

\section{ABSORPTION PROCESSES}

The primary absorption process in ablation interactions is inverse bremsstrahlung absorption. This process occurs when an electron in the Coulomb field of an ion absorbs an incident photon. The absorption coefficient $K_{i b}$ is given by ${ }^{6 ; 7}$

$$
K_{i b}=8 \sqrt{\pi}\left(\frac{e^{2}}{4 \pi \epsilon_{0}}\right)^{3} \frac{4}{3 c^{3}} \frac{Z_{i}^{2}}{m_{e}^{2}} n_{e} n_{i} \sqrt{\frac{m_{e}}{2 k_{B} T}}\left(\frac{\pi^{2} c^{2}}{\hbar \omega^{3}}\right)\left[1-\exp \left(-\frac{\hbar \omega}{k_{B} T}\right)\right]
$$

where $n_{e}$ is the electron density, $n_{i}$ and $Z_{i}$ the are the density and ionization of the ion species respectively, $\omega$ is the laser angular frequency, and $T$ is the plasma temperature. All other symbols have their usual meanings. 
Note that the exponential term $\left[1-\exp \left(-\hbar \omega / k_{B} T\right)\right]$ is included to account for photon production by stimulated emission. Crucially, the absorption coefficient is proportional to $n_{e} n_{i} Z_{i}^{2}$, indicating a significant impact from the population distribution.

The reduction in wavelength increases the photon energy above the threshold for photo-ionization to occur. The expression for the photo-ionization absorption coefficient $K_{p i}$ is given simply by

$$
K_{p i}=\sigma_{b f} n_{i}
$$

where $\sigma_{b f}$ is the photo-ionization cross-section, and can be taken from the literature ${ }^{8}$. Alternatively, the photoionization cross-section can be calculated using a similar treatment to equation $1^{7}$;

$$
\sigma_{p i}=\frac{16}{3 \pi} \frac{g_{i+1}}{g_{i}} \alpha^{3} \frac{\pi^{2} c^{2}}{\hbar \omega^{3}}\left[\frac{2 R_{d} Z_{i}^{4}}{n^{3}}\right]
$$

where $R_{d}$ is the Rydberg energy in Joules, $n$ is the principal quantum number of the ionizing state, and $\alpha$ is the fine structure constant given by

$$
\alpha=\frac{e^{2}}{4 \pi \epsilon_{0} \hbar c} .
$$

Whilst the photo-ionization cross-section is dependent only on material properties, the species density for the eligible ions will vary across the simulation. The ionization model chosen to relate the temperature, electron density, and ion population distribution of the ablated plasma will therefore be a significant choice.

\section{IONIZATION MODELS}

\subsection{The Saha-Boltzmann model}

The Saha-Boltzmann model ${ }^{9}$ is the standard model used to calculate ionization balance within a plasma. The principal expression for this model gives the ratio between a charge state $Z_{i}$ with density $n_{i}$ and the level above $\left(Z_{i}+1\right)$ with density $n_{i+1}$ as

$$
\frac{n_{i+1}}{n_{i}}=\frac{g_{i+1}}{g_{i}} \frac{2}{n_{e} \Lambda^{3}} \exp \left(-\frac{E_{i o n}}{k_{B} T}\right)
$$

where $\Lambda$ is the thermal de Broglie wavelength, given by

$$
\Lambda=\frac{h}{\sqrt{2 \pi m_{e} k_{B} T}} .
$$

The Saha-Boltzmann ratio is a tried and tested piece of physics, its only drawback is the premise on which it is based; that the plasma be in equilibrium. In the case of a laser-produced plasma this is an approximation.

\subsection{Accounting for perturbation by photo-ionization}

The Saha-Boltzmann ratio considers the two levels it relates to be in equilibrium with each other. In the case when the lower level is effected by photo-ionization, the balance of processes that achieves this equilibrium must be adjusted. Assuming the pulse time of the laser is on order of nanoseconds, we can conclude equilibrium is reached and add photo-ionization to the list of processes we consider.

In an unperturbed plasma, the collisional processes that pertain to changes in ionization are the collisional ionization rate and the three-body recombination rate. The collisional ionization rate is the rate at which electron-ion collisions result in ionization of the ion. The rate of ionization $K_{c i}$ of a given level $i$ in units of $\mathrm{cm}^{3} \mathrm{~s}^{-1}$ is given by the Lotz approximation ${ }^{10}$

$$
K_{c i}=3 \times 10^{-6} \frac{g_{i}}{\left(k_{B} T\right)^{3 / 2}} \frac{I_{e}(y)}{y}\left[\mathrm{~cm}^{3} \mathrm{~s}^{-1}\right]
$$

where $k_{B} T$ is in electron volts, $I_{e}(y)$ is the exponential integral

$$
I_{e}(y)=\int_{y}^{\infty} \frac{1}{x} e^{-x} d x
$$


and $y$ is the reduced ionization energy, given by $y=E_{\text {ion }} / k_{B} T$.

The rate coefficient $K_{3 b r}$ for the reverse process, three-body recombination, can be found by use of detailed balance and the Saha-Boltzmann ratio ${ }^{7}$. In equilibrium $K_{c i}$ and $K_{3 b r}$ will be balanced like so;

$$
n_{i} n_{e} K_{c i}=n_{(i+1)} n_{e} K_{3 b r}
$$

After some rearrangement, we can replace the ratio $n_{i} / n_{(i+1)}$ with equation 5 to find

$$
K_{3 b r}=\frac{g_{i}}{g_{(i+1)}} \frac{n_{e} \Lambda^{3}}{2} \exp \left(\frac{E_{i}}{k_{B} T}\right) K_{c i}
$$

Figure 1a shows a three level atomic system where these processes are in balance.

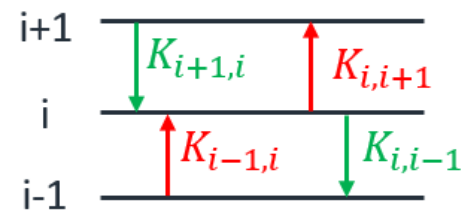

(a)

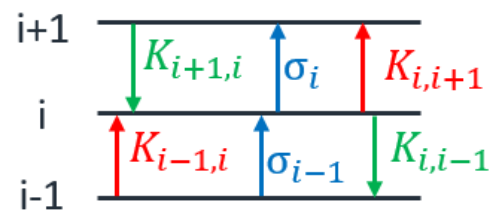

(b)

Figure 1: Example 3-level systems showing the populating and de-populating processes for an ionization level $i$ both (a) without photo-ionization and (b) with photo-ionization.

In a plasma driven by a laser with photon energy above the photo-ionization threshold, we can add photoionization into equation 9 to give a new level dependent balance between the populating and de-populating processes - see figure 1b. For example, in the case where photo-ionization is possible only for the neutral and the $1+$ ion we have:

$$
\begin{aligned}
\text { for } i=0: & n_{0}\left(n_{e} K_{0,1}+\sigma_{0,1} \Phi\right) & =n_{1} n_{e} K_{1,0} \\
\text { for } i=1: & n_{1}\left(n_{e} K_{1,0}+n_{e} K_{1,2}+\sigma_{1,2} \Phi\right) & =n_{0}\left(n_{e} K_{0,1}+\sigma_{0,1} \Phi\right)+n_{2} n_{e} K_{2,1} \\
\text { for } i=2: & n_{2}\left(n_{e} K_{2,1}+n_{e} K_{2,3}\right) & =n_{1}\left(n_{e} K_{1,2}+\sigma_{1,2} \Phi\right)+n_{3} n_{e} K_{3,2} \\
\text { for } i>2: & n_{i}\left(n_{e} K_{i,(i-1)}+n_{e} K_{i,(i+1)}\right) & =n_{(i-1)} n_{e} K_{(i-1), i}+n_{(i+1)} n_{e} K_{(i+1), i} \\
\text { and for } i_{\text {max }}: & n_{i} n_{e} K_{i,(i-1)} & =n_{(i-1)} n_{e} K_{(i-1), i} .
\end{aligned}
$$

where $\Phi$ is the incident radiation flux.

\subsection{Continuum lowering}

In the high density limit, the Saha-Boltzmann model loses accuracy due to the introduction of perturbative effects. One such effect is continuum lowering, where the ionization potential of the ions is reduced by the close proximity of other ion potentials. This effect has been quantified by Stewart and Pyatt ${ }^{11}$, who give a correction factor to the ionization such that

$$
\frac{\Delta E_{i o n}}{E_{\text {ion }}}=\frac{a_{0}}{Z+1}\left(\frac{2}{a_{C}}\right)
$$

The distance $a_{C}$ is given by either the Debye length $\lambda_{D}$, or the ion sphere radius $r_{0}$ given by

$$
\frac{4 \pi}{3} r_{0}^{3}=\frac{1}{n_{i}} .
$$

The choice that gives the smallest correction should be chosen as the model reconciles two previous suggested models; the ion-sphere model and the Debye-Hückel model ${ }^{12}$ (the former being applicable in the low temperature, high density limit and the latter applicable in the high temperature, low density limit). There are other models 
that predict the effects of continuum lowering, the most notable being that of Ecker and Kröll ${ }^{13}$, however the Stewart-Pyatt model was selected as it is widely utilized and is applicable in our high density, low temperature limit. This continuum lowering correction can also be applied to the rate-equation model. We need not modify the model any further, but should include any transitions that have become possible with the reduction in ionization energy.

\section{TIME-RESOLVED CALCULATIONS}

\subsection{Reference values}

The first step towards producing time-resolved measurements was producing a set of reference curves to relate temperatures to energy content values. These energy content values were calculated by simply adding the energy in the free electrons and ions together:

$$
U_{i}=\frac{3}{2} n_{e} k_{B} T_{e}+\sum_{i=1}^{i} n_{i} E_{i}
$$

This dependence on the ion populations $n_{i}$, which are calculated by an iterative process, makes calculating $U_{i}$ from $T_{e}$ relatively simple, but this is not the case for the converse. This is why reference curves have been implemented, to allow simple transitions between the temperature and energy content.

\subsubsection{Saha-Boltzmann vs. rate equation}

Initially, reference curves for the Saha-Boltzmann and rate equation models were produced for comparison. As the population ratios are dependent on both the plasma temperature and electron density, an iterative algorithm must be implemented. The population distribution for each temperature was calculated first using a trial electron density. The projected ion densities were normalized such that the total species density was equal to the solid density and a projected electron density was extracted from these values. The difference between the projected electron density and the initial density was then halved and added to the lower of the two, and the process was repeated until the correction required was below a chosen tolerance (the bisection method). In the case of the rate equation model, this is complicated slightly by the contribution of the $\mathrm{n}_{(i+2)}$ level to the calculation of the $\mathrm{n}_{(i+1)}$ level. To rectify this, a second iteration is included. For each trial electron density, the population
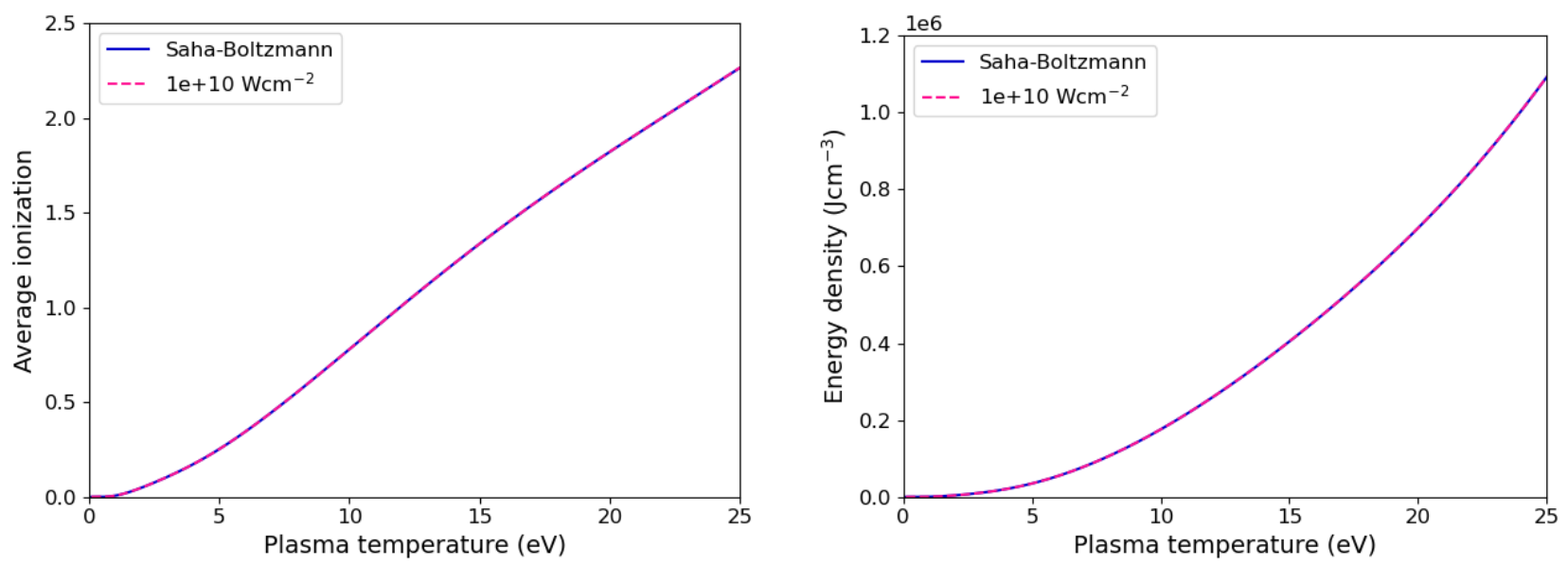

Figure 2: Average ionization and energy density of aluminium as a function of temperature for the Saha-Boltzmann and rate equation models with an irradiance of $1 \times 10^{10} \mathrm{~W} \mathrm{~cm}^{-2}$.

distribution is calculated using trial populations for $\mathrm{n}_{(i+2)}$ first, and then by the populations just predicted. 

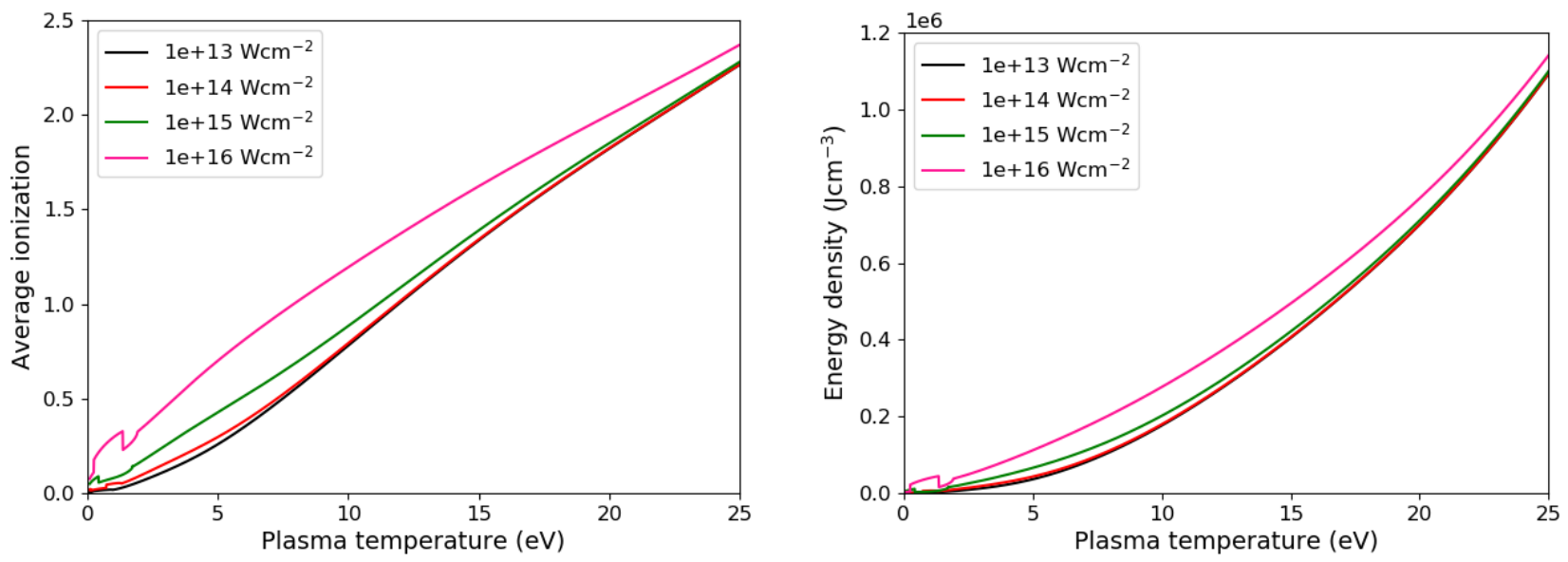

Figure 3: Average ionization and energy density of aluminium as a function of plasma temperature for the rate equation model at irradiances from $1 \times 10^{13} \mathrm{~W} \mathrm{~cm}^{-2}$ to $1 \times 10^{16} \mathrm{~W} \mathrm{~cm}^{-2}$.

This is repeated until the change in the populations is below a given tolerance, and the outer iteration over the electron density can progress.

Initial focus was on irradiances in the region of those seen experimentally $3 ; 4 ; 5$ and the difference between the two models will increase with irradiance, so a comparison at the peak irradiance of $\approx 1 \times 10^{10} \mathrm{~W} \mathrm{~cm}^{-2}$ was conducted first, shown in figure 2. At these low irradiances, the two models overlap perfectly, with negligible contribution from photo-ionization. This implies that for the irradiances seen in experiment the rate equation model is applicable but offers no improvement over the Saha-Boltzmann model.

At higher irradiances, we see the differences in the models increase - see figure 3 - however significant changes are not seen below $1 \times 10^{15} \mathrm{~W} \mathrm{~cm}^{-2}$. Significant deviation from the Saha-Boltzmann model at these temperatures indicates the plasma is no longer in equilibrium. Irregularities in the continuity of the higher irradiance curves are likely caused by instability in the iteration method.

\subsubsection{The effects of continuum lowering}
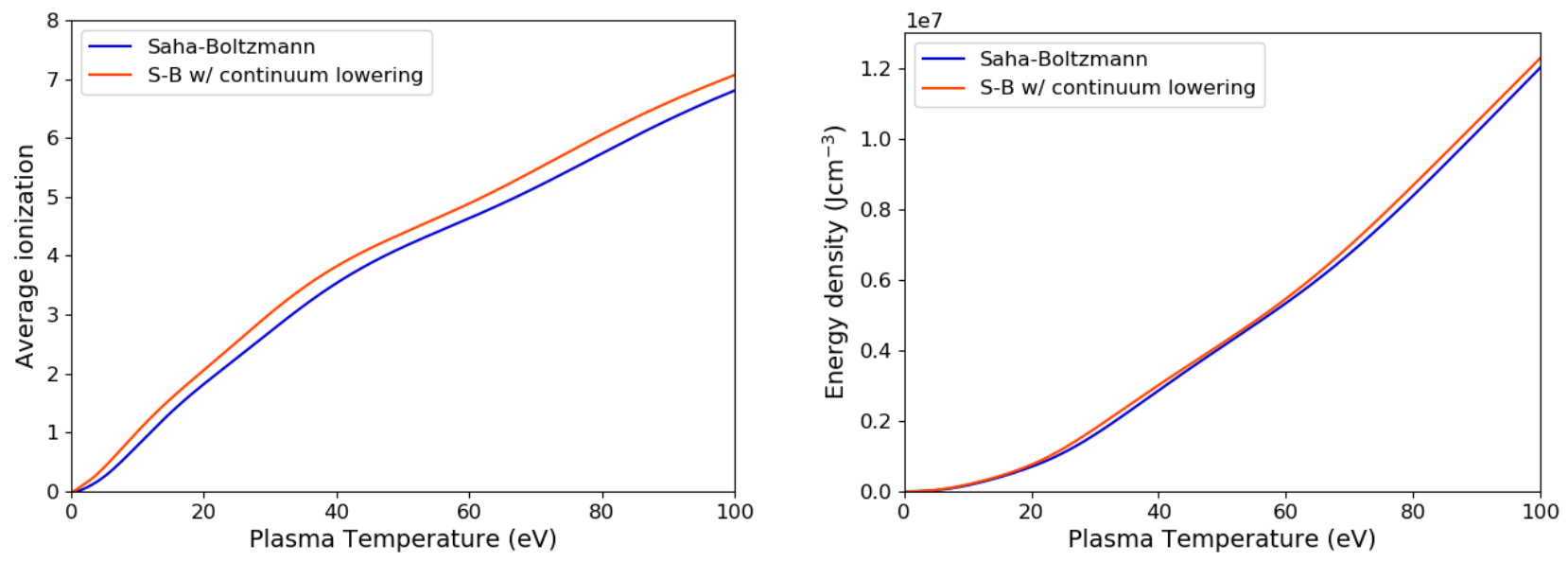

Figure 4: Average ionization and energy density of aluminium as a function of plasma temperature for the SahaBoltzmann model with and without the Stewart-Pyatt ionization energy correction. 
As with the rate equation model, reference curves for the Saha-Boltzmann model and a corrected form using the Stewart-Pyatt model were produced. These are shown in figure 4. The difference in the two models is relatively small, peaking at around 0.2 for $Z_{i}$, but the impact this will have on time-resolved measurements is unclear. Figure 5 shows the effect on the absorption coefficients. This is more significant as $K_{i b}$ is proportional to

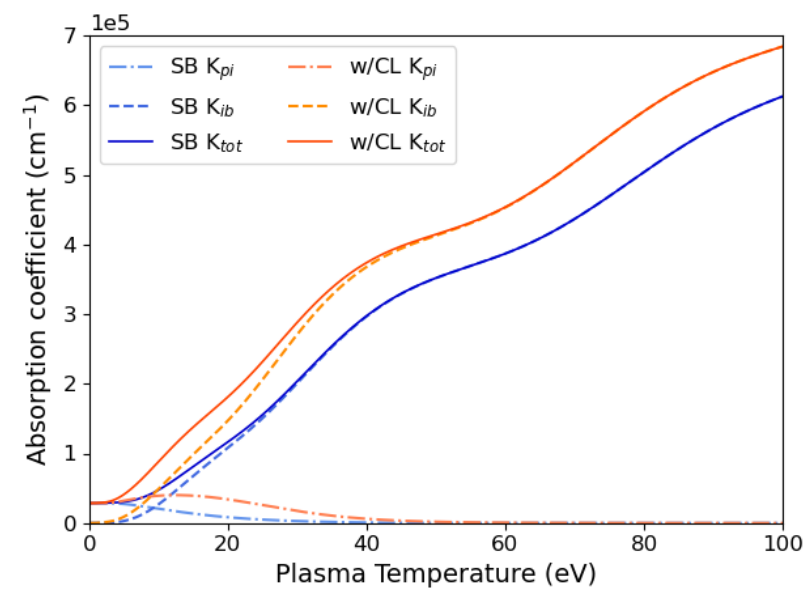

Figure 5: Absorption coefficients for inverse bremsstrahlung $K_{i b}$, photo-ionization $K_{p i}$, and the combination of the two $K_{\text {tot }}$ against plasma temperature for both the corrected (w/CL) and uncorrected (SB) Saha-Boltzmann model in aluminium.

$n_{e} n_{i} Z_{i}^{2}$ and $K_{p i}$ will now have contributions from higher ionization levels. This will result in more rapid heating of the target. The point at which photo-ionization drops below $10 \%$ of the total absorption shifts significantly from $18.5 \mathrm{eV}$ to $26.7 \mathrm{eV}$ but $K_{i b}$ dominates from $10 \mathrm{eV}$ regardless.

\subsubsection{Continuum lowering in the rate equation model}

The effects of continuum lowering on the rate equation model have also been investigated. The same algorithm was implemented, with the ionization energies corrected using the Stewart-Pyatt model. The results for $1 \times 10^{10} \mathrm{~W} \mathrm{~cm}^{-2}$ are shown in figure 6. The correction has affected the Saha-Boltzmann and rate equation models
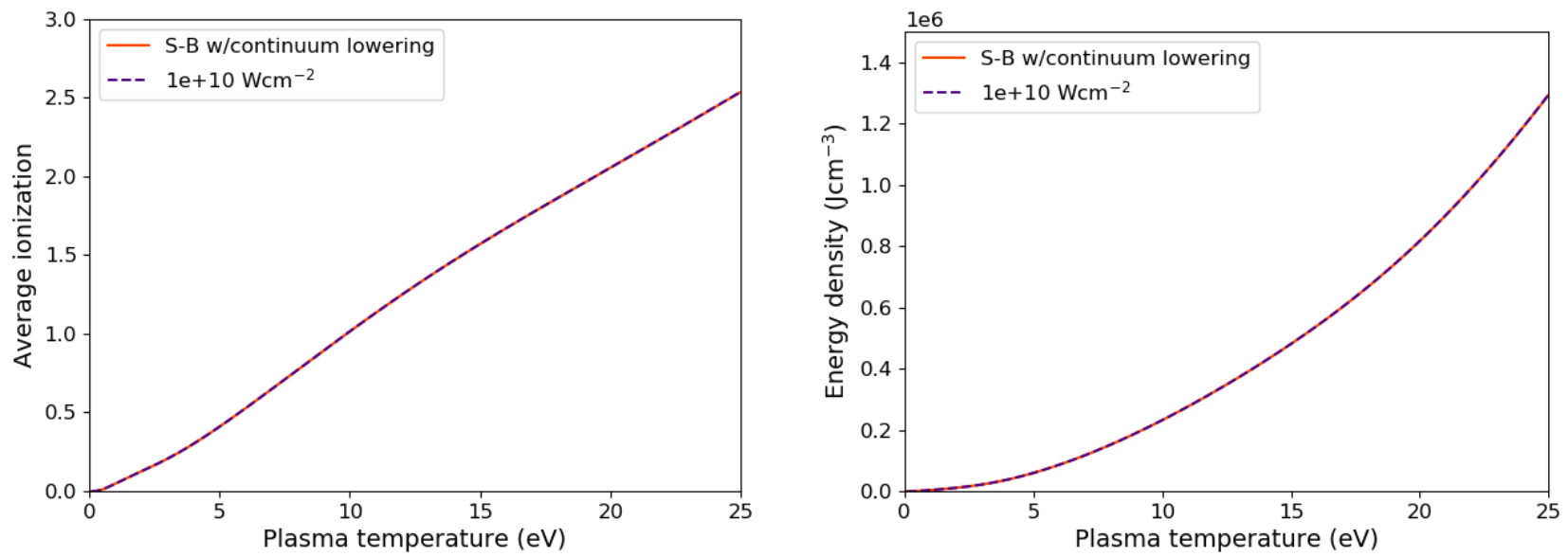

Figure 6: Average ionization and energy density of aluminium as a function of plasma temperature for the StewartPyatt corrected Saha-Boltzmann and rate equation models with an irradiance of $1 \times 10^{10} \mathrm{~W} \mathrm{~cm}^{-2}$. 
equally, as would be expected, generating the same consistency between the models as before the correction.

Results for multiple higher irradiances are shown in figure 7 . The effect of continuum lowering is present for all irradiances, with reduced impact at higher irradiances.
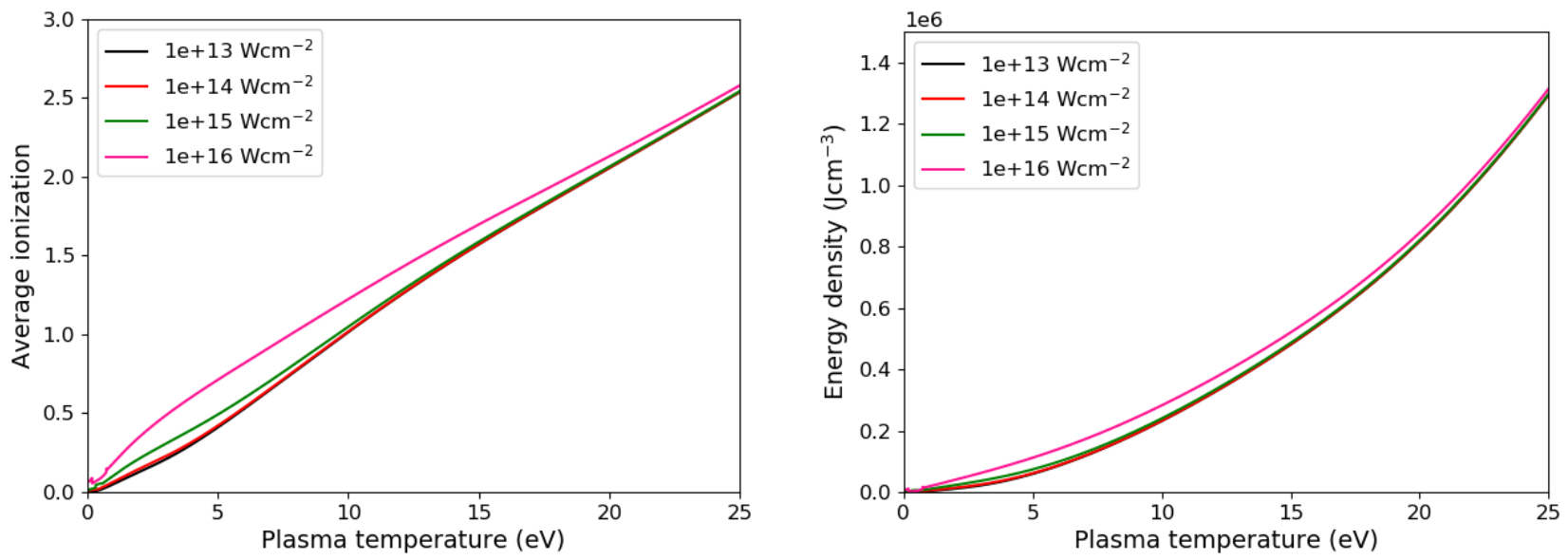

Figure 7: Average ionization and energy density of aluminium as a function of plasma temperature for the StewartPyatt corrected rate equation model at irradiances from $1 \times 10^{13} \mathrm{~W} \mathrm{~cm}^{-2}$ to $1 \times 10^{16} \mathrm{~W} \mathrm{~cm}^{-2}$.

\subsection{Single cell variant}

Using the models detailed in section 3, a simple time-resolved scenario was constructed. A $10 \mathrm{~nm}$ slice of material, of unit area, was irradiated with time-dependent pulses of $46.9 \mathrm{~nm}$ radiation. The cell was assigned a temperature and energy content, and absorption coefficients were calculated accordingly. These were then used to calculate the reduction in intensity across the cell, and therefore the deposited energy. Finally, the energy content was updated and compared to the reference values to find the new cell temperature. This was repeated for each time
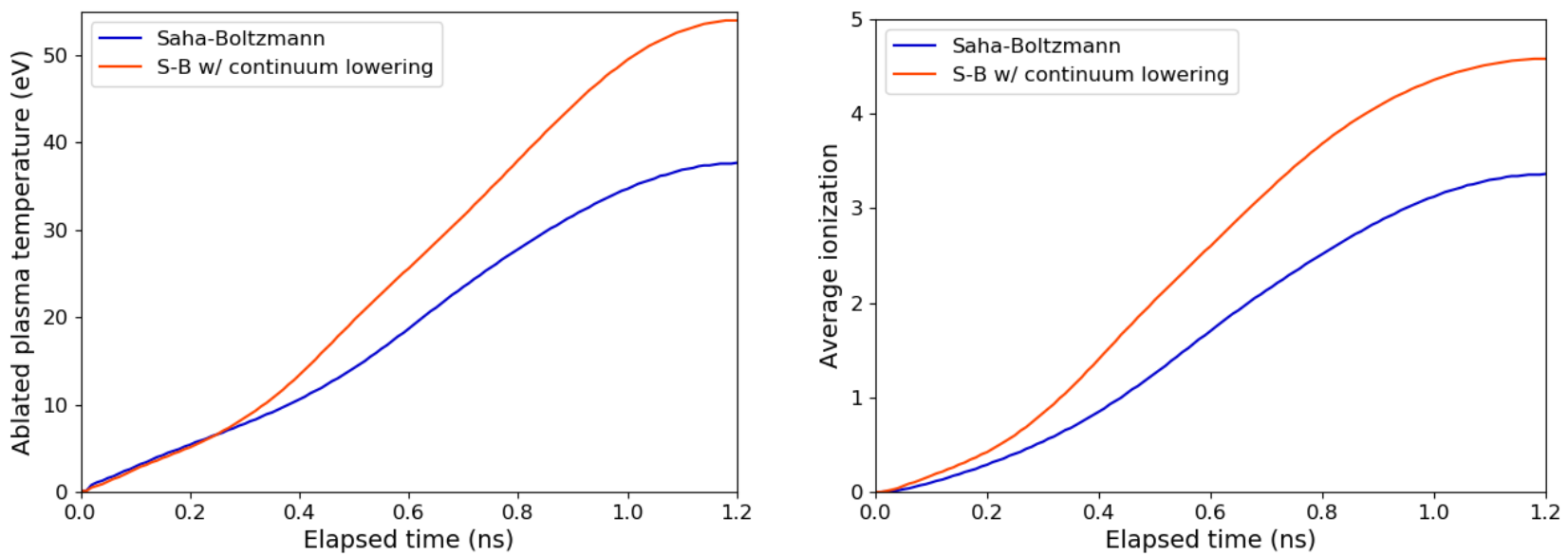

Figure 8: Temporal evolution of the plasma temperature and average ionization of a $10 \mathrm{~nm}$ aluminium cell for the uncorrected and Stewart-Pyatt corrected Saha-Boltzmann model.

step with the next irradiance value in the time-dependent pulse. As there were no calculations performed in each time step, it was trivial to run both the Saha-Boltzmann model and the Stewart-Pyatt correction simultaneously. 
A sine-squared pulse of $1.2 \mathrm{~ns}$ duration with a peak irradiance of $3 \times 10^{10} \mathrm{~W} \mathrm{~cm}^{-2}$ was modelled initially, again to best match experimental parameters. The predicted temperatures and ionizations for both models are shown in figure 8. The small changes introduced by continuum lowering in temperature and ionization, married with the larger change in the absorption coefficient, have led to a significant increase in the final temperature, from $37.7 \mathrm{eV}$ to $54.0 \mathrm{eV}$, and ionization, from 3.36 to 4.48 , of the test cell. This is likely to have strong implications in the spatially-resolved simulations.

\subsection{Density variations}

Thus far the effects of temperature on the various models have been well considered, but the density has remained constant at $\mathrm{n}_{\text {solid }}$. The spatially-resolved simulations in section 4.4 are hydrostatic and do not consider plasma expansion, so the effects of density are key to determining the validity of these results. As a simple test, a cell at $10 \%$ of $\mathrm{n}_{\text {solid }}$ was examined under the same conditions as before, the results of which are shown in figure 9 .
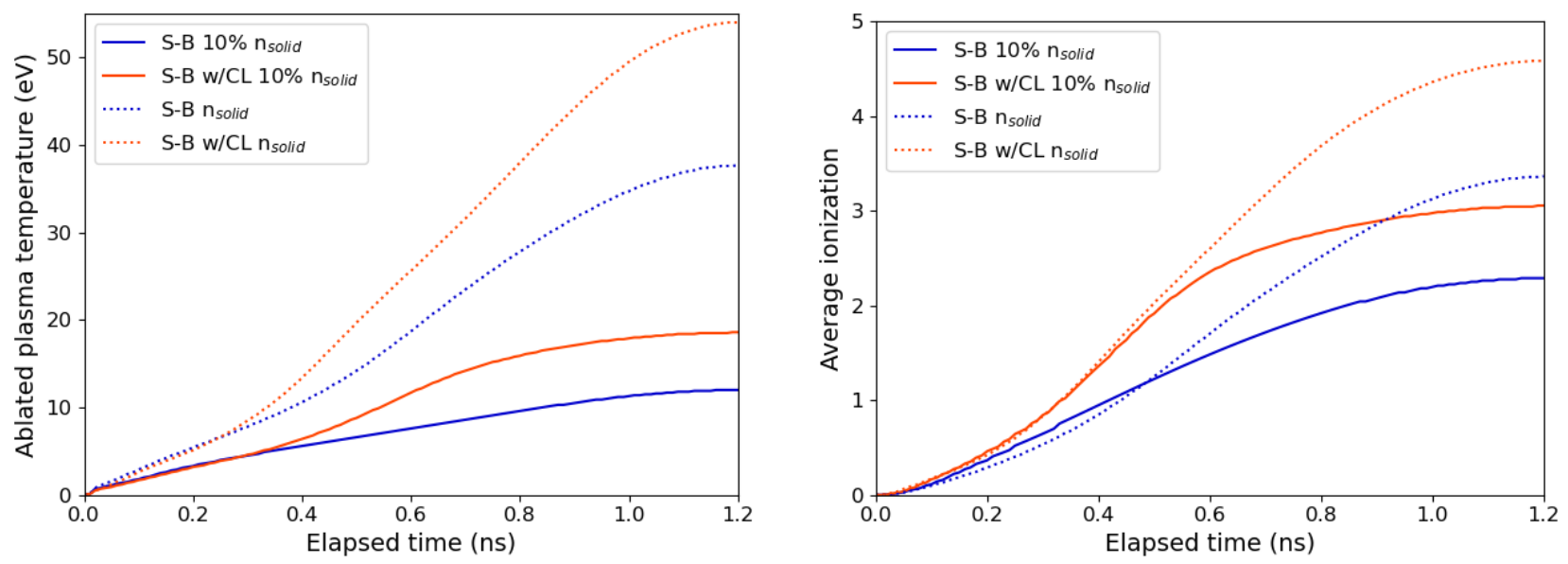

Figure 9: Temporal evolution of the plasma temperature and average ionization of a $10 \mathrm{~nm}$ aluminium cell at solid density and at $10 \%$ solid density for the uncorrected and Stewart-Pyatt corrected Saha-Boltzmann model.

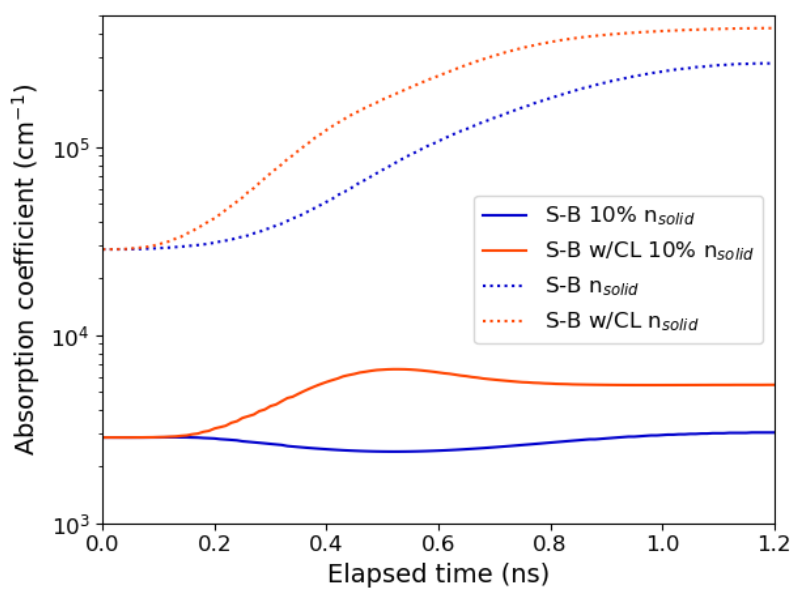

Figure 10: Temporal evolution of the total absorption coefficient of a $10 \mathrm{~nm}$ aluminium cell at solid density and at $10 \%$ solid density for the uncorrected and Stewart-Pyatt corrected Saha-Boltzmann model.

The decrease in density has reduced both the final temperature and the final ionization for both models as 
expected. The most significant change however, is in the absorption coefficient - shown in figure 10 . The total absorption coefficient for the plasma has reduced by an order of magnitude at early times and by nearly two at late times. This indicates that in the case of an expanding plasma, the lower density plume will absorb little of the incoming radiation and energy deposition will be dominated by the material at close to solid density. From this, we can conclude that the hydrostatic model has a good chance of being valid, with the plume contributing little to the absorption. These low density results are also of interest in the ionization of gas jet targets by extreme ultraviolet laser pulses.

\subsection{Multi cell variant}

Expanding the simulations to include spatial resolution was simple. The algorithm for each cell remained largely unchanged, but an additional calculation of the irradiance reaching each cell was added between them. This was calculated from the energy deposited in the previous cell. An example temperature distribution is shown in figure 11a. By varying the peak irradiance and integrating over the pulse we can produce a graph of fluence plotted against ablated depth - see figure 11b. Determining how to measure the ablation depth requires some

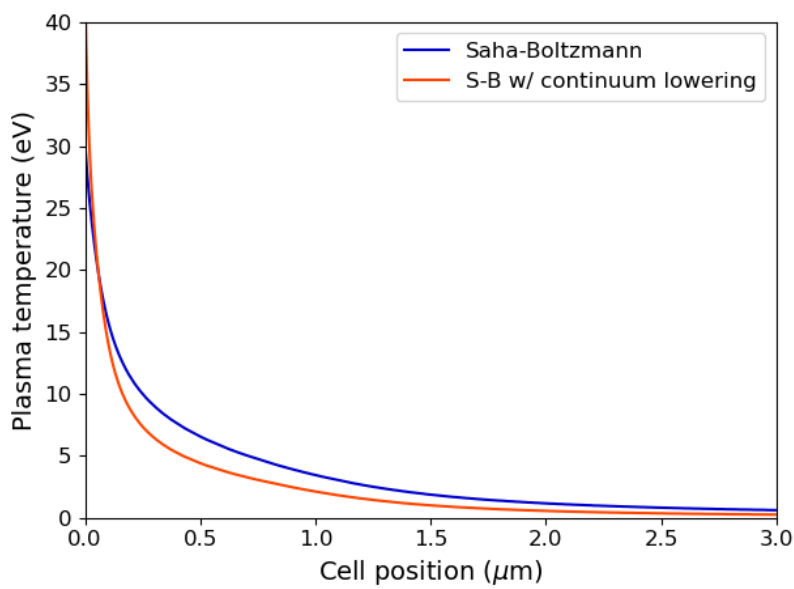

(a)

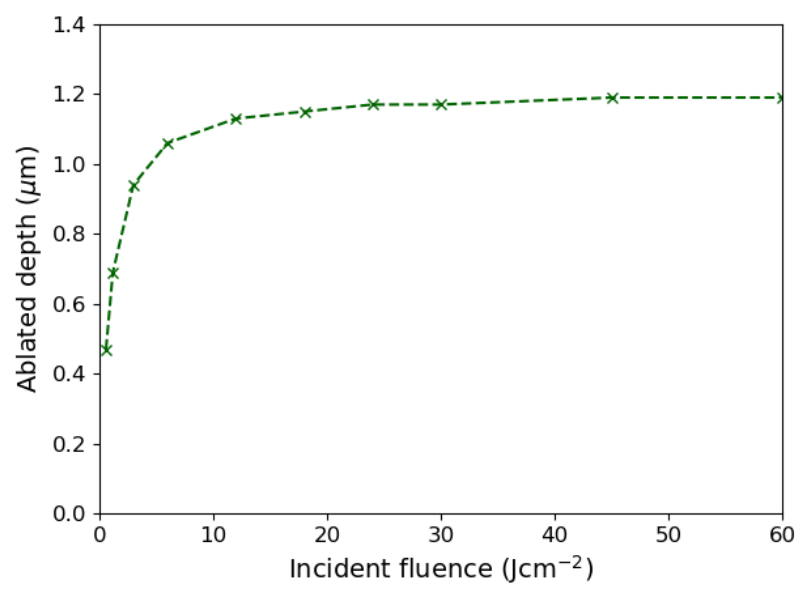

(b)

Figure 11: (a) Spatial distribution of plasma temperature for a peak irradiance of $3 \times 10^{10} \mathrm{~W} \mathrm{~cm}^{-2}$ and (b) predicted ablated depths as a function of incident fluence for an aluminium target.

thought. The simplest choice is a minimum plasma temperature or minimum ionization; melting temperature, boiling temperature, and $10 \%$ ionization are sensible choices. A minimum ionization was selected as the most likely condition for a complete solid-plasma transition.

Two main conclusions present themselves from figure 11b; there appears to be a maximum achievable ablation depth, and the ablated depth approaches this value rapidly (below $10 \mathrm{~J} \mathrm{~cm}^{-2}$ ).

\section{CONCLUSIONS}

The high photon energies of extreme-ultraviolet photons activate photo-ionization as a contributing absorption process in laser ablation. The absorption coefficients for inverse bremsstrahlung and photo-ionization in an aluminium plasma at a variety of temperatures and at multiple densities have been calculated. The rate equation model showed no deviation in ionization or energy content from the Saha-Boltzmann model (corrected or uncorrected) at irradiances below $1 \times 10^{13} \mathrm{~W} \mathrm{~cm}^{-2}$, and no significant deviation below $1 \times 10^{15} \mathrm{~W} \mathrm{~cm}^{-2}$. Accounting for continuum lowering created a small deviation from the Saha-Boltzmann model in average ionization and energy density but this manifested as a larger change in the absorption coefficient. In the case of both ionization models, inverse bremsstrahlung dominates the absorption process above temperatures of $\approx 10 \mathrm{eV}$. 
These calculated values have then been used to formulate a simple temporally-resolved algorithm simulating a $10 \mathrm{~nm}$ slice of target irradiated by $1.2 \mathrm{~ns}$ pulse of $46.9 \mathrm{~nm}$ radiation. These simulations showed more significant effects on the final temperature and ionization of the cell due to continuum lowering. Investigating the absorption coefficients at $10 \%$ solid density showed a one to two order of magnitude reduction in absorption coefficient, implying that the majority of laser absorption occurs at close to solid density. Expanding this to a spatiallyresolved model generated a fluence to ablation depth relationship with saturation-like behavior.

\section{References}

[1] Benware, B. R., Macchietto, C. D., Moreno, C. H., and Rocca, J. J., "Demonstration of a High Average Power Tabletop Soft X-Ray Laser," Physical Review Letters 81(26), 5804 (1998).

[2] Rocca, J. J. et al., "Capillary discharge tabletop soft X-ray lasers reach new wavelengths and applications," Comptes rendus de l'Académie des Sciences, Série IV 1, 1065 (2000).

[3] Aslanyan, V. et al., "Ablation and transmission of thin solid targets irradiated by intense extreme ultraviolet laser radiation," APL Photonics 1, 066101 (2016).

[4] Juha, L. et al., "Ablation of organic polymers by 46.9-nm-laser radiation," Applied Physics Letters 86(3), 034109 (2005).

[5] Benware, B. R. et al., "Focusing of tabletop soft-x-ray laser beam and laser ablation," Optics Letters 24(23), 1714 (1999).

[6] Hutchinson, I., [Principles of Plasma Diagnostics] (2005).

[7] Tallents, G. J., [An Introduction to the Atomic and Radiation Physics of Plasmas], Cambridge University Press (2018).

[8] Reilman, R. F. and Manson, S. T., "Photoabsorption cross sections for positive atomic ions with Z $\leq 30$," The Astrophysical Journal Supplement Series 40, 815 (1979).

[9] Saha, M. N., "LIII. Ionization in the solar chromosphere," Philosophical Magazine, Series 6 40(238), 472 (1920).

[10] Lotz, W., "An empirical formula for the electron-impact ionization cross-section," Zeitschrift für Physik 206, 205 (1967).

[11] Stewart, J. C. and Pyatt, K. D., "Lowering of ionization potentials in plasmas," The Astrophysical Journal 144, 1203 (1966).

[12] Debye, P. and Huckel, E., "Zur Theorie der Elektrolyte. I. Gefrierpunktserniedrigung und verwandte Erscheinungen," Physikalische Zeitschrift 24, 185 (1923).

[13] Ecker, G. and Kröll, W., "Lowering of the ionization energy for a plasma in thermodynamic equilibrium," Physics of Fluids 6(1), 62 (1963). 